\title{
Spin Thermoelectric Effects in a Strongly Correlated Double Quantum Dot System
}

\author{
E. KARWACKI* \\ Faculty of Physics, Adam Mickiewicz University, 61-614 Poznań Poland
}

\begin{abstract}
Spin thermoelectric effects through a strongly correlated double quantum dot system embedded in a AharonovBohm ring coupled to two leads with Rashba spin-orbit coupling have been investigated theoretically by means of the finite-U slave-boson technique. This method provides a reliable description of electron transport through quantum dot systems in the low temperature regime $T \ll T_{\mathrm{K}}$, where $T_{\mathrm{K}}$ is the so-called Kondo temperature. Particular emphasis is put on the possibility of the so-called spin Seebeck effect in such a system as a more sensitive probe of the Kondo state.
\end{abstract}

DOI: 10.12693 /APhysPolA.127.478

PACS: 73.23.-b, 73.63.Kv, 72.15.Qm, 85.35.Ds

With the recent developments in the field of spin caloritronics $[1,2]$ one of the main goals remains finding effective methods to control the thermally-induced spin current. Since the quantum dot systems present unique properties such as a narrow density of states [3] and relatively easy control over parameters such as gate voltage or the possibility for tuning the quantum interference effects, the interest in such systems, as a possible thermoelectric and spin thermoelectric devices, has grown. However, the aforementioned unique properties alone are insufficient for spin thermoelectric effects to arise. The basic methods used to induce spin thermovoltage between the dots have mostly relied on using ferromagnetic leads [4-6]. However, it is interesting to examine electrically controlled Rashba spin-orbit coupling (RSO) $[7,8]$ induced spin thermoelectric effects in a double quantum dot (DQD) structure which can be realized in an Aharonov-Bohm (AB) ring setup [9]. We show that the spin thermoelectric effects in transport through such a system are significantly modified in the Kondo regime, to which end we employ slave-boson method for finite- $U$ Hamiltonian [10].

The system considered in this paper consists of two single-level $\varepsilon_{i}$ quantum dots with onsite Coulomb interaction $U$ described by the Hamiltonian $H_{d q d}=$ $\sum_{i \sigma} \varepsilon_{i} d_{i \sigma}^{\dagger} d_{i \sigma}+U \sum_{i} n_{i \uparrow} n_{i \downarrow}$ and parallelly coupled to two reservoirs $\beta=L, R$ of electrons with wavevector $k$ and spin $\sigma=\uparrow, \downarrow$ described by the Hamiltonian $H_{e}=\sum_{\boldsymbol{k} \beta \sigma} \varepsilon_{\boldsymbol{k} \beta} c_{\boldsymbol{k} \beta \sigma}^{\dagger} c_{\boldsymbol{k} \beta \sigma}$. Hamiltonian describing tunneling between the $\mathrm{i}$-th dot and electrode $\beta$ takes the form $H_{t}=\sum_{\boldsymbol{k} \beta \sigma i}\left(V_{\boldsymbol{k} \beta \sigma i}^{\prime} c_{\boldsymbol{k} \beta \sigma}^{\dagger} d_{i \sigma}+\right.$ H.c. $)$. The properties of the Aharonov-Bohm ring with Rashba spinorbit coupling between the dots and the electrodes are taken into consideration in the tunneling amplitudes as $V_{\boldsymbol{k} \beta \sigma i}^{\prime}=V_{\boldsymbol{k} \beta \sigma i} \exp \left(\hat{\beta} \hat{\alpha} i \phi_{\sigma} / 4\right)$, where $\phi_{\sigma}=\phi+\hat{\sigma} \phi_{R}$ is the sum of AB phase $\phi$ and RSO phase $\phi_{R}$, while $\hat{\sigma}= \pm 1$

* corresponding author; e-mail: karwacki@amu.edu.pl (for $\sigma=\uparrow, \downarrow), \hat{\beta}=\delta_{\beta L}-\delta_{\beta R}$ and $\hat{\alpha}=\delta_{i 2}-\delta_{i 1}$ with $\delta_{x y}$ being the Kronecker's delta.

To describe the strongly correlated Kondo regime a method of slave-bosons with finite- $\mathrm{U}$ is used [10], where the initial creation $d_{i \sigma}^{\dagger}$ (annihilation $d_{i \sigma}$ ) operators of both dots are exchanged with superposition of pseudofermion and boson operators $f_{i \sigma}^{\dagger} z_{i \sigma}^{\dagger}\left(z_{i \sigma} f_{i \sigma}\right)$. Operator $z_{i \sigma}^{\dagger}=e_{i} p_{i \sigma}^{\dagger}+p_{i \bar{\sigma}} d_{i}^{\dagger}$ acts as a projection operator on the extended Fock space of the dot. We assume henceforth saddle-point approximation for the slave-boson operators, which allows for the use of mean values of the slaveboson operators $\left\langle z_{i \sigma}^{(\dagger)}\right\rangle \equiv z_{i \sigma},\left\langle p_{i \sigma}^{(\dagger)}\right\rangle \equiv p_{i \sigma},\left\langle e_{i}^{(\dagger)}\right\rangle \equiv e_{i}$, and $\left\langle d_{i}^{(\dagger)}\right\rangle \equiv d_{i}$. In addition, it is necessary to introduce boundary conditions, such as the conservation of states $e_{i}^{2}+\sum_{\sigma} p_{i \sigma}^{2}+d_{i}^{2}=1$ and the conservation of charge $p_{i \sigma}^{2}+d_{i}^{2}=\left\langle f_{i \sigma}^{\dagger} f_{i \sigma}\right\rangle$.

After the transformation, the double dot Hamiltonian takes the form $\tilde{H}_{d q d}=\sum_{i \sigma} \tilde{\varepsilon}_{i \sigma} f_{i \sigma}^{\dagger} f_{i \sigma}$, where $\tilde{\varepsilon}_{i \sigma}=$ $\varepsilon_{i}+\lambda_{i \sigma}^{(2)}$. The tunneling Hamiltonian can now be written down as $\tilde{H}_{t}=\sum_{\boldsymbol{k} \beta \sigma i}\left(\tilde{V}_{\boldsymbol{k} \beta \sigma i}^{\prime} c_{\boldsymbol{k} \beta \sigma}^{\dagger} f_{i \sigma}+\right.$ H.c. $)$, where $\tilde{V}_{\boldsymbol{k} \beta \sigma i}^{\prime}=z_{i \sigma} V_{\boldsymbol{k} \beta \sigma i}^{\prime}$. This parameter is used later on to introduce coupling coefficient $\tilde{\Gamma}_{i j \sigma}=2 \pi \tilde{V}_{\boldsymbol{k} \beta \sigma i}^{\prime} \tilde{V}_{\boldsymbol{k} \beta \sigma j}^{*} \rho_{\beta}$, where $\rho_{\beta}$ is the density of states in the electrode $\beta$. The constraints introduced above and the Coulomb correlations reside in the additional term $E_{s b}=\sum_{i}\left[U d_{i}^{2}+\right.$ $\left.\lambda_{i}^{(1)}\left(e_{i}^{2}+\sum_{\sigma} p_{i \sigma}^{2}+d_{i}^{2}-1\right)-\sum_{\sigma} \lambda_{i \sigma}^{(2)}\left(p_{i \sigma}^{2}+d_{i}^{2}\right)\right]$, where $\lambda_{i}^{(1)}$ and $\lambda_{i \sigma}^{(2)}$ are Lagrange multipliers. The transformation leads to an effective Hamiltonian $H_{e f f}=\tilde{H}_{d q d}+$ $\tilde{H}_{t}+H_{e}+E_{s b}$.

Mean values of the slave-boson parameters necessary for the calculation of transport parameters are derived via self-consistent equations using Hellmann-Feynman theorem for the effective Hamiltonian $\partial_{\chi_{i}} H_{\text {eff }}=0$, where $\chi_{i}=\left(\lambda_{i}^{(1)}, \lambda_{i \sigma}^{(2)}, e_{i}, p_{i \sigma}, d_{i}\right)$ :

$$
e_{i}^{2}+\sum_{\sigma} p_{i \sigma}^{2}+d_{i}^{2}-1=0
$$




$$
\begin{aligned}
& p_{i \sigma}^{2}+d_{i}^{2}-K_{0, i, \sigma}=0, \\
& \sum_{\sigma} \partial_{e_{i}} \ln z_{i \sigma} K_{1, i, \sigma}+\lambda_{i}^{(1)} e_{i}=0, \\
& \sum_{\sigma} \partial_{p_{i \sigma^{\prime}}} \ln z_{i \sigma} K_{1, i, \sigma}+\left(\lambda_{i}^{(1)}-\lambda_{i \sigma^{\prime}}^{(2)}\right) p_{i \sigma^{\prime}}=0, \\
& \sum_{\sigma} \partial_{d_{i}} \ln z_{i \sigma} K_{1, i, \sigma}+\left(U+\lambda_{i}^{(1)}-\sum_{\sigma} \lambda_{i \sigma}^{(2)}\right) d_{i}=0,
\end{aligned}
$$

where for $k=0,1, K_{k, i, \sigma}=(1 / 2 \pi i) \int d \varepsilon\left(\varepsilon-\tilde{\varepsilon}_{\sigma}\right)^{k} G_{i i, \sigma}^{<}$. Here $G_{i i, \sigma}^{<}$are the diagonal elements of lesser Green's function matrix $\boldsymbol{G}_{\sigma}^{<}=i \boldsymbol{G}_{\sigma}^{r}\left(f_{L} \tilde{\boldsymbol{\Gamma}}_{L \sigma}+f_{R} \tilde{\boldsymbol{\Gamma}}_{R \sigma}\right) \boldsymbol{G}_{\sigma}^{a}$, where $f_{L(R)}$ is the Fermi-Dirac distribution for the left and right electrode and symbols $\boldsymbol{G}_{\sigma}^{r}=\left(\boldsymbol{G}_{\sigma}^{a}\right)^{\dagger}$ denote retarded and advanced Green's functions, which are derived using equation of motion technique [11].

The coupling coefficient matrix takes the form

$$
\tilde{\boldsymbol{\Gamma}}_{\beta \sigma}=\left(\begin{array}{cc}
z_{1 \sigma}^{2} & q z_{1 \sigma} z_{2 \sigma} \mathrm{e}^{-\hat{\beta} \mathrm{i} \frac{\phi \sigma}{2}} \\
q z_{1 \sigma} z_{2 \sigma} \mathrm{e}^{\hat{\beta} \mathrm{i} \frac{\phi \sigma}{2}} & z_{2 \sigma}^{2}
\end{array}\right) \Gamma_{\beta} .
$$

Here we assume that the coupling of the dots to the electrodes is symmetric, i.e., $\Gamma_{L}=\Gamma_{R}=\Gamma$. Parameter $q \in\langle 0,1\rangle$ describes the strength of the interference effects due to the intermediate electron states between the dots and the electrodes [12].

To calculate spin thermoelectric parameters we assume linear response regime, which leads to wellknown expressions for spin conductance $G_{s}=$ $(e \hbar / 2) \sum_{\sigma} \hat{\sigma} L_{0 \sigma}$, electronic contribution to thermal conductance $\kappa_{e l}=(1 / T) \sum_{\sigma}\left(L_{2 \sigma}-L_{1 \sigma}^{2} / L_{0 \sigma}\right)$, spin thermopower $S_{s}=-(1 / 2|e| T) \sum_{\sigma} \hat{\sigma} L_{1 \sigma} / L_{0 \sigma}$ and spin figure of merit $Z_{s} T=\left|G_{s}\right| S_{s}^{2} T / \kappa_{e l}$. Here $L_{n=0,1,2, \sigma}=$ $(1 / h) \int d \varepsilon(\varepsilon-\mu)^{n}\left(-\partial_{\varepsilon} f\right)_{T, \mu} T_{\sigma}(\varepsilon)$ is the Onsager transport coefficient which relates currents to the driving forces and $T_{\sigma}(\varepsilon)=\operatorname{Tr}\left(\boldsymbol{G}_{\sigma}^{a} \tilde{\boldsymbol{\Gamma}}_{R \sigma} \boldsymbol{G}_{\sigma}^{r} \tilde{\boldsymbol{\Gamma}}_{L \sigma}\right)$ is the transmission coefficient of electron with spin $\sigma$.

Generally in multilevel systems, such as a double quantum dot system, one can expect the emergence of discrete interference-induced states, either bonding and anti-bonding or super- and subradiant due to the interaction of the electrons tunneling through both of the dots via intermediate states in the electrodes [13]. This fact is particularly emphasized in full width at half maximum of local density of states (LDOS) $\rho_{i \sigma}=-(1 / \pi) \operatorname{Im} G_{i \sigma \sigma}^{r}$ which in the Aharonov-Bohm-Rashba setup is additionally spin-dependent

$$
\Gamma_{\sigma}^{e f f, \pm}=z_{\sigma}^{2} \Gamma\left(1 \pm q \cos \phi_{\sigma}\right),
$$

where the upper index \pm denotes one of the interferenceinduced states. This function plays important role in determining the so-called Kondo temperature of the system.

Apart from the effective magnetic field $B_{e f f}$, spin thermoelectric effects can be observed as well, as presented in Fig. 2. Fig. 2a and c presents spin thermopower as a function of $\mathrm{AB}$ phase $\phi$. It is evident that absence of
From the analysis of the above introduced expression it is evident, that for strongest interference, i.e., $q=1$ and $\phi_{\sigma}=n \pi$, where $n=\ldots,-2,-1,0,1,2, \ldots$ one of the widths tends to zero and thus LDOS is singular. On the other hand for $\phi_{\sigma}=(n-1 / 2) \pi$ the width becomes insensitive to the changes in the parameter $q$.

In the strongly correlated regime, spin accumulation, induced in the electrodes due to the RSO, leads to different renormalization of the quantum dots' spin-dependent energy levels, i.e., $\varepsilon_{\sigma}=\varepsilon_{d}+\lambda_{\sigma}^{(2)}$. The non-zero energy difference can be understood as an effective magnetic field $B_{\text {eff }}=\varepsilon_{\uparrow}-\varepsilon_{\downarrow}=\lambda_{\uparrow}^{(2)}-\lambda_{\downarrow}^{(2)}$ [14]. Changes of this field are plotted in Fig. 1 as a function of AB phase $\phi$ for different values of the RSO induced phase $\phi_{R}$ and dots' bare energy levels $\varepsilon_{d}=-U / 2$ and $\varepsilon_{d}=-U / 4$. Firstly, there is a noticeable difference in magnitude of ca. $10^{-3}$ between the $B_{\text {eff }}$ values for dots' energy levels $\varepsilon_{d}=-U / 2$ and $\varepsilon_{d}=-U / 4$. For both cases the effective magnetic field $B_{\text {eff }}$ vanishes for $\phi=n \pi$, where $n=-4,-3, \ldots, 3,4$. For the case of $\phi_{R}=\pi / 4$ presented in Fig. $1 \mathrm{~b}$ the maximal values of the field appear for $\phi=(2 n+11 / 25) \pi$ whereas the minimal values for $\phi=(2 n+1+14 / 25) \pi$, while the opposite is true for the case of $\phi_{R}=7 \pi / 4$. For the case of $\phi_{R}=3 \pi / 4$ the maxima and minima are slightly shifted and appear for $\phi=(2 n+14 / 25) \pi$ and $\phi=(2 n+11 / 25) \pi$ respectively. This discussion also holds true for Fig. 1a after noticing that here the field is of opposite sign, i.e., $B_{e f f}\left(\varepsilon_{d}=-U / 2\right) \cdot B_{e f f}\left(\varepsilon_{d}=-U / 4\right)<0$.

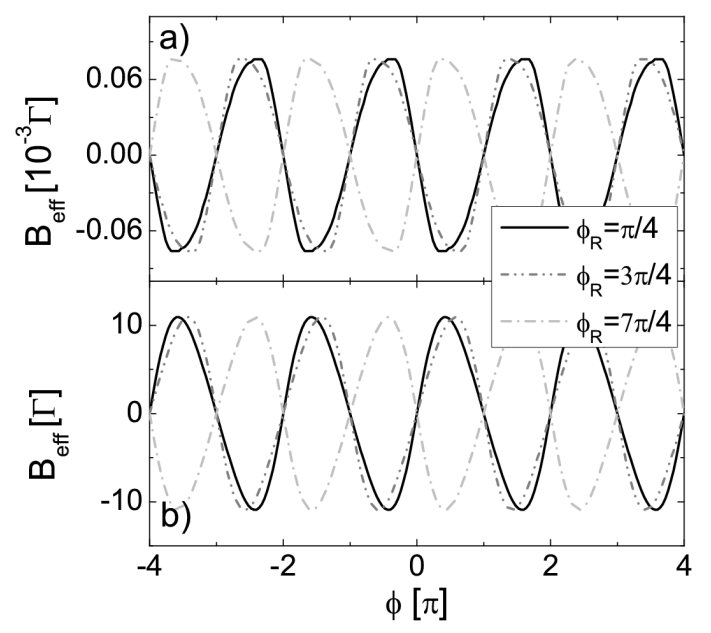

Fig. 1. Effective magnetic field $B_{\text {eff }}$ as a function of AB phase $\phi$ for different values of RSO phase $\phi_{R}$ and dots' energy level $\varepsilon_{d}=-U / 2 \mathrm{a}$ ) and $\left.\varepsilon_{d}=-U / 4 \mathrm{~b}\right)$. The other parameters: $q=0.95, T=0.001 \Gamma, U=6 \Gamma$.

spin thermopower coincides with zero effective magnetic field $B_{\text {eff }}$, i.e., $\phi=n \pi$. For $\varepsilon_{d}=-U / 2$ maximal spin thermopower is smaller than for $\varepsilon_{d}=-U / 4$ and peaks are less pronounced. Similiarly to the plot of effective 

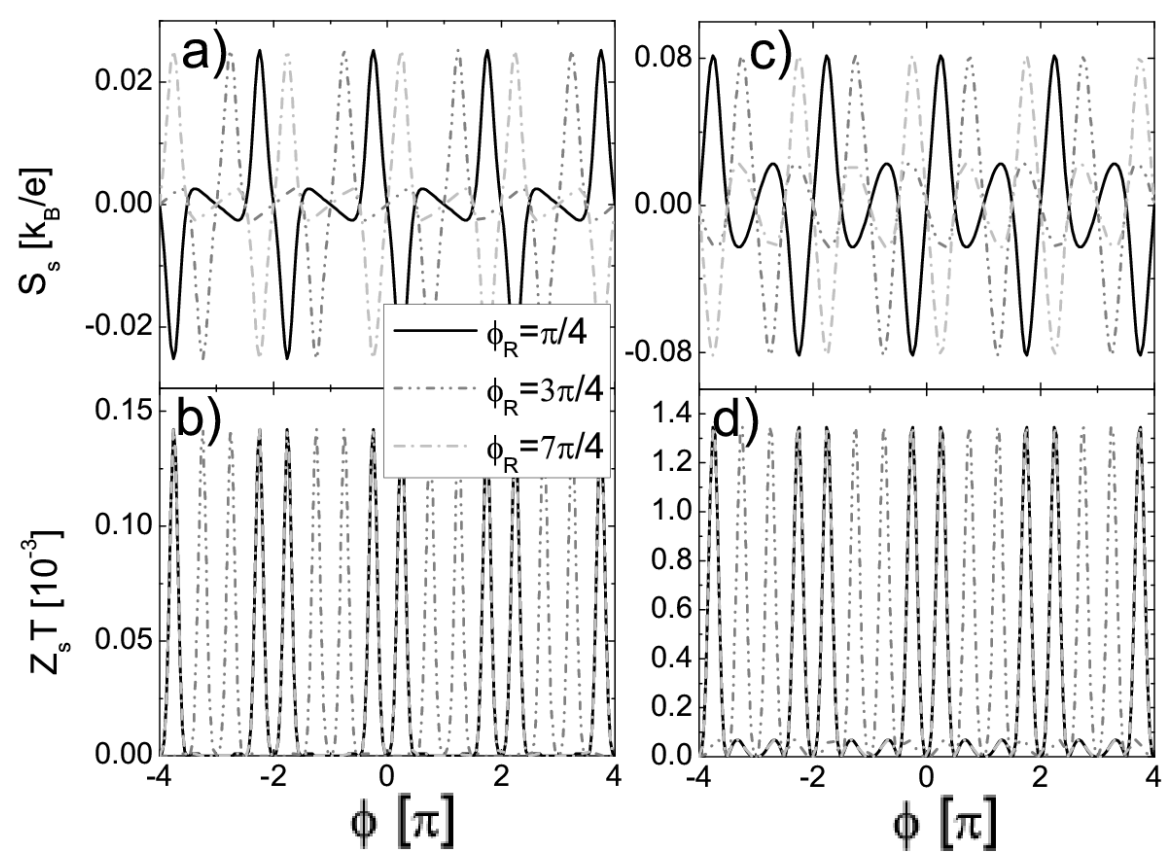

Fig. 2. Spin thermopower $S_{s}$ a), c) and spin figure of merit $Z_{s} T$ b), d) as a function of AB phase $\phi$ for different values of RSO phase $\phi_{R}$ and dots' energy level $\left.\left.\varepsilon_{d}=-U / 2 \mathrm{a}\right), \mathrm{b}\right)$ and $\left.\varepsilon_{d}=-U / 4 \mathrm{c}\right)$, d). The other parameters: $q=0.95$, $T=0.001 \Gamma, U=6 \Gamma$.

magnetic field, both spin Seebeck coefficients are of opposite sign, i.e., $S_{s}\left(\varepsilon_{d}=-U / 2\right) \cdot S_{s}\left(\varepsilon_{d}=-U / 4\right)<0$. For RSO induced phase $\phi_{R}=\pi / 4$ and $\phi_{R}=7 \pi / 4 S_{s}$ are opposite, while for $\phi_{R}=3 \pi / 4$ the maxima and minima are shifted.

Fig. $2 \mathrm{~b}$ and $\mathrm{d}$ presents spin figure of merit as a function of $\mathrm{AB}$ phase $\phi$. Thermoelectric efficency vanishes for values of $\phi$ for which the spin thermopower $S_{s}$ vanished. The appearance of extremal values is consistent with the maxima and minima of spin thermopower $S_{s}$. For RSO induced phases $\phi_{R}=\pi / 4$ and $\phi_{R}=7 \pi / 4$ the respective spin figure of merit functions are indistinguishable, whereas for $\phi_{R}=3 \pi / 4$ maximal values are shifted as in the plot of spin thermopower. The compensation of effective magnetic field in the electron-hole symmetry point $\varepsilon=-U / 2$ leads to smaller values of spin figure of merit than in the case of asymmetric position of the level $\varepsilon_{d}=-U / 4$.

In conclusion we have shown that spin thermoelectric effects can be induced in a strongly correlated double quantum dot system electrically due to Rashba spin-orbit coupling. Spin accumulation induced by this interaction generates an effective magnetic field that splits dots' energy levels and generates a non-zero spin polarization of the quantum dots. This in turn leads to the enhancement of spin thermopower and spin figure of merit.

\section{Acknowledgments}

This work was supported by the National Science Center in Poland as the project No. DEC2012/04/A/ST3/00372. The author would also like to thank Piotr Trocha for suggesting the problem and pro- viding helpful comments that aided in improving the manuscript.

\section{References}

[1] K. Uchida, S. Takahashi, K. Harii, J. Ieda, W. Koshibae, K. Ando, S. Maekawa, and E. Saitoh, $\mathrm{Na}$ ture 455, 778 (2008).

[2] G.E.W. Bauer, E. Saitoh, B.J. van Wees, Nature Mater. 11, 391 (2012).

[3] G.D. Mahan, J.O. Sofo, Proc. Natl. Acad. Sci. USA 93, 7436 (1996).

[4] P. Trocha, J. Barnaś, Phys. Rev. B 85, 085408 (2012).

[5] R. Świrkowicz, M. Wierzbicki, J. Barnaś, Phys. Rev. B 80, 195409 (2009).

[6] I. Weymann, J. Barnaś, Phys. Rev. B 88, 085313 (2013).

[7] Q.-F. Sun, J. Wang, H. Guo, Phys. Rev. B 71, 165310 (2005).

[8] Ł. Karwacki, P. Trocha, J. Barnaś, J. Phys.: Condens. Matter 25, 505305 (2013).

[9] Y.S. Liu, X.K. Hong, J.F. Feng, X.F. Yang, Nanoscale Res. Lett. 6, 618 (2011).

[10] G. Kotliar, A.E. Rückenstein, Phys. Rev. Lett. 57, 1362 (1986).

[11] H. Haug, A.-P. Jauho, Quantum kinetics and optics of Semiconductors, Springer Verlag, Berlin 1996.

[12] P. Trocha, J. Barnaś, Phys. Rev. B 76, 165432 (2007).

[13] P. Trocha, J. Phys.: Condens. Matter 24, 055303 (2012).

[14] J.S. Lim, M. Crisan, D. Sánchez, R. Lòpez, I. Grosu, Phys. Rev. B 81, 235309 (2010). 